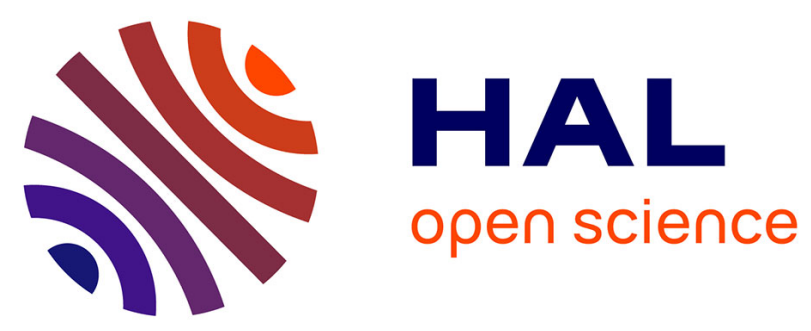

\title{
Assessing Extrinsic Membrane Protein Dependency to PI4P Using a Plasma Membrane to Endosome Relocalization Transient Assay in Nicotiana benthamiana
}

Mehdi Doumane, Marie-Cécile Caillaud

\section{To cite this version:}

Mehdi Doumane, Marie-Cécile Caillaud. Assessing Extrinsic Membrane Protein Dependency to PI4P Using a Plasma Membrane to Endosome Relocalization Transient Assay in Nicotiana benthamiana. Marisa S. Otegui. Plant Endosomes Methods and Protocols, 2177, Springer, pp.95-108, 2020, 978-10716-0766-4. 10.1007/978-1-0716-0767-1_9. hal-03004339

\section{HAL Id: hal-03004339 \\ https://hal.science/hal-03004339}

Submitted on 18 Oct 2021

HAL is a multi-disciplinary open access archive for the deposit and dissemination of scientific research documents, whether they are published or not. The documents may come from teaching and research institutions in France or abroad, or from public or private research centers.
L'archive ouverte pluridisciplinaire $\mathbf{H A L}$, est destinée au dépôt et à la diffusion de documents scientifiques de niveau recherche, publiés ou non, émanant des établissements d'enseignement et de recherche français ou étrangers, des laboratoires publics ou privés. 


\title{
Assessing extrinsic membrane protein dependency to PI4P using a PM to endosome re-localisation transient assay in Nicotiana benthamiana
}

\author{
Mehdi Doumane and Marie-Cécile Caillaud \\ Laboratoire Reproduction et Développement des Plantes, Université de Lyon, ENS de Lyon, \\ UCB Lyon 1, CNRS, INRA, F-69342 Lyon, France.
}

Corresponding author: marie-cecile.caillaud@ens-lyon.fr

\begin{abstract}
Phosphoinositides are key players from which the various membranes of the cells acquire their identity. The relative accumulation of these low abundant anionic phospholipids in the cytosolic leaflet of the plasma membrane and of various endomembranes generates a landmark code, responsible for the selective recruitments of extrinsic proteins at given membranes. One of the key players in the protein/lipid interaction at the plasma membrane in plant cells, is the phosphatidylinositol 4-phosphate (PI4P), which patterns the recruitment of effector proteins from the plasma membrane to organelles along the endocytic pathway. Here we propose a fast assay to assess the requirement of PI4P for membrane localisation of extrinsic membrane proteins in vivo. This system relies on perturbing PI4P distribution in plant cells via the action of a PI4P phosphatase that depletes the pool of PI4P at a given membrane. This system efficiently decreases PI4P levels, and can therefore be easily used to assess requirement of PI4P (and electrostatics) for the targeting of extrinsic membrane proteins to the plasma membrane or endosomes. Ultimately, this system could also be extended to test the phosphatase activity in planta of enzymes putatively involved in PI4P metabolism.
\end{abstract}

\section{Key words}

Anionic lipids, Phosphoinositides, fluorescent-tagged protein, membrane proteins, phosphatase assay, membrane trafficking, plasma membrane, electrostatics, agrobacterium transformation.

\section{Introduction}

Biological membranes act as selectively permeable borders separating two compartments. For instance the plasma membrane (PM) limits cytoplasm and extracellular space. That way, the PM allows physico-chemical parameters to be different inside the cell compared to the outer medium, facilitating or even allowing metabolic reaction to occur (Monnard \& Walde, 2015).

In some Prokaryotes and in Eukaryotes, additional biological membranes are present in the cells. These endomembrane structures define intracellular compartments in which additional conditions, different from cytoplasm, can exist: the lumen of the endoplasmic reticulum is an oxidising 
environment whereas the cytoplasm is reducing; the lumen of the vacuole is acid whereas cytosol is neutral, and so on.

Lipids and proteins compose biological membranes, which properties come from both intrinsic properties of these components, and of emergent ones generated by their assembly at the supramolecular scale. Membrane lipids are amphipathic molecules self-organising in bilayer of two leaflets. Each leaflet exposes polar groups toward water solvent and interacting with water molecules or other lipid polar groups. On the other hand, proteins can be associated to membranes via various means. Intrinsic proteins possess hydrophobic domains, such as hydrophobic alpha helix, embedded inside the membranes. Extrinsic proteins are associated with membranes, either by a covalent bound, or by more labile non-covalent bounds they make with membrane lipids or proteins (Platre \& Jaillais, 2017).

Interestingly, extrinsic proteins that associate to the cytosolic leaflet of a membrane appear to be targeted mainly or exclusively to a given membrane. For example, SidM protein from Legionella pneumophila localises only to the membrane surrounding this intracellular bacteria when infecting Mammalian cells (Zhu et al., 2010, Brombacher et al., 2009, Del Campo et al., 2014) whereas Arabidopsis MEMBRANE ASSOCIATED REPRESSOR KINASE 2 (MARK2) protein localises to the plasma membrane (Simon et al., 2016). How is this selective subcellular association to membrane achieved? SidM directly interacts with the phosphatidylinositol-4-phosphate (PI4P), a lipid present on the membrane it localises to, and MAKR2 is targeted to the highly electronegative electrostatic field beneath the plasma membrane, partially driven by PI4P (Simon et al., 2016, Platre et al., 2018)

Phosphoinositides, including PI4P, are indeed key players from which the various membranes of the cells acquire their identity. These low abundant membrane lipids derive from phosphatidic acid (PA, the simplest phospholipid) bearing in addition an inositol head that can be virtually phosphorylated at three different positions. In plants, so far only phophatidylinositol (PI), three phosphatidylinositolphosphate (PI3P, PI4P, and PI5P) and two phosphatidylinositol-bisphosphates $\left[\mathrm{PI}(3,5) \mathrm{P}_{2}\right.$ and $\left.\mathrm{PI}(4,5) \mathrm{P}_{2}\right]$ were reported. The differential accumulation of theses lipids in the cytosolic leaflet of the plasma membrane and of various endomembrane generates a landmark code, responsible for the selective recruitments of extrinsic proteins at given membranes (Lemmon, 2008, Noack \& Jaillais, 2017). All together, these recruitments at the molecular level can occur by direct binding to a cognate lipid like SidM does, and/or via the recognition of lipid induced biophysical properties such as membrane curvature, local electrostatic field or packing defects (Platre \& Jaillais, 2017, Lemmon, 2008).

We generated a system that allows the targeting at the plasma membrane - or at both plasma membrane and endosomes - of yeast SUPPRESSOR OF ACTIN 1 (Sac1) PI4P-4phosphtase domain (Sac12-518; figure 1A)(Noack et al., 2019, Guo et al., 1999, Platre et al., 2018, Simon et al., 2016). In this system, a catalytically dead version of Sac1 phosphatase domain ( $\mathrm{Sac}^{\mathrm{C} 392 \mathrm{~S}}{ }_{2-518}$ in which a catalytic cysteine was mutated into a serine) serves as negative control (figure $1 \mathrm{~A}$ ). The $\mathrm{N}$-terminal myristoylation and palmitoylation sequence (MAP) was added to the construct in order to target the catalytic domain of the phosphatase at the plasma membrane. The internal fluorescent protein (later on called FP) allows the detection of $N$. benthamiana cells transiently expressing MAP-FP-Sac1 $1_{2-518}$ and confirmed that MAP-FP-Sac1 $1_{2-518}$ recombinant proteins localise at the plasma membrane. In some occasions, depending on the level of expression, MAP-FP-Sac $1_{2-518}$ could also be observed both at the plasma membrane and endosomes, which allowed us to test the role of both pools of PI4P on the localisation of the protein of interest (figure 1B). We confirmed the efficiency and specificity of the 
system toward PI4P by coexpressing MAP-FP-Sac1 $1_{2-518}$ together with anionic lipids biosensors. PI4P biosensors, composed of a fluorescent protein (FP) fused to a PI4P lipid-binding domain, allow to dynamically track PI4P (Figure 1C;(Hammond et al., 2014, Simon et al., 2014, Simon et al., 2016). For instance, mCITRINE-P4M $\mathrm{M}^{\mathrm{SidM}}$ binds to PI4P and highlights PI4P major pool that sits at the plasma membrane in plant cells (figure 1D and G). When co-expressed with MAP-FP-Sac1 $1_{2-518}$, mCITRINE$\mathrm{P}_{4} \mathrm{M}^{\mathrm{SidM}}$ relocalises to endosomes where the minor pool of PI4P sits in plant cells (Simon et al., 2014). Depending on the level of expression of the MAP-FP-Sac1 ${ }_{2-518}$, mCITRINE-P4M $\mathrm{M}^{\mathrm{SidM}}$ could solubilise into the cytoplasm when both pool of PI4P, at the plasma membrane and in the endosomes, are depleted (figure $1 \mathrm{E}, \mathrm{F}$ and $\mathrm{H}$ ). Consistently, MAP-FP-Sac ${ }^{\mathrm{C} 392 \mathrm{~S}}{ }_{2-518}$ does not cause mCITIRNE-P4M $\mathrm{M}^{\mathrm{SidM}}$ delocalisation (figure 1I).

This system efficiently decreases PI4P levels, and can therefore be easily used to assess requirement of PI4P for the targeting of extrinsic membrane proteins to the plasma membrane or endosomes. We generated mTURQUOISE2 (mTU2, blue) and mCHERRY $(\mathrm{mCH}$, red) fluorescent MAP-FP-Sac12-518 and MAP-FP-Sac ${ }^{\mathrm{C} 392 \mathrm{~S}}{ }_{2-518}$ recombinant-protein. We as well used mTU2, mCITRINE (mCIT, yellow) and red fluorescent PI4P sensors (Simon et al., 2016, Simon et al., 2014) in order to obtain an extended toolbox. Therefore, it is possible to transiently express in $N$. benthamiana a fluorescent protein of interest to be tested together with MAP-FP-Sac1 $1_{2-518}$ and the PI4P biosensor as internal control (see 3. Methods, and Table 1). Ultimately, this system could also be extended to test the phosphatase activity in planta of enzymes supposedly important for the metabolism of PI4P.

As proof of concept, we successfully co-expressed MAP-mTU2-Sac1 $1_{2-518}$ and mCITRINE-P4M ${ }^{\text {SidM }}$ and 2xmCHERRY-KA1 ${ }^{\text {MARK1 }}$ (figure 2;(Platre et al., 2018). KA1 domain from human MARK1 protein corresponds to a cationic stretch targeting the protein to the plasma membrane via electrostatic interactions (Moravcevic et al., 2010). PI4P being the main anionic lipids responsible for electronegativity of the PM in plants, KA1 ${ }^{\text {MARK1 }}$ localisation at the plasma membrane of plant cells relies on PI4P (Simon et al., 2016). 2xmCHERRY-KA1 MARK1 localisation was unchanged when coexpressed with MAP-mTU2-Sac1 ${ }^{\mathrm{C} 392 \mathrm{~S}}$ (figure $2 \mathrm{~A}, \mathrm{~B}, \mathrm{C}$, and D). On the other hand, 2xmCHERRYKA1 $1^{\text {MARK1 }}$ delocalised from the plasma membrane upon MAP-mTU2-Sac1 $1_{2-518}$ induced PI4Pdepletion, and co-localised with also delocalised mCITRINE-P4M ${ }^{\text {SidM }}$ (figure 2E, F, and G; (Simon et al., 2016).

\section{Materials}

\subsection{Plant and Agrobacteria Material}

1. Three to five weeks-old Nicotiana benthamiana plants, before vegetative to reproductive transition - no flower buds or flowers formed yet.

2. GV3101 Agrobacterium tumefaciens Strain: Genotype C58 (rifampicin genomic resistance) Ti pMP90 (pTiC58DT-DNA; gentamycin resistant).

\subsection{Culture Media and Stock Solutions}

1. Lysogeny Broth (LB) liquid medium: $10 \mathrm{~g} / \mathrm{l}$ peptone, $5 \mathrm{~g} / \mathrm{l}$ Yeast Extract, $5 \mathrm{~g} / \mathrm{l}(86 \mu \mathrm{M}) \mathrm{NaCl}$.

2. LB plates: LB liquid medium supplemented with $14 \mathrm{~g} / \mathrm{l}(\sim 1,4 \%)$ agar.

3. Yeast Extract Broth (YEB) plates: $5 \mathrm{~g} / \mathrm{l}$ beef extract, $1 \mathrm{~g} / \mathrm{l}$ yeast extract, $5 \mathrm{~g} / \mathrm{l}$ peptone, $(5 \mathrm{~g} / \mathrm{l}$ $(41 \mu \mathrm{M})$ sucrose, $14 \mathrm{~g} / \mathrm{l}(\sim 1,4 \%)$ agar, $\mathrm{pH} 7.2$, supplemented to $2 \mathrm{mM} \mathrm{MgSO}$ (or $\left.\mathrm{MgCl}_{2}\right)$ after autoclave. 


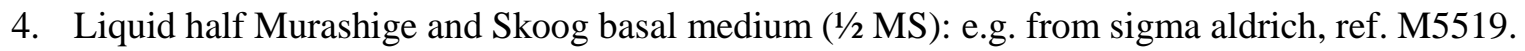

5. Stock solution of $1 \mathrm{M} \mathrm{MgCl}_{2}$.

6. Stock solution of $1 \mathrm{M}$ Tris- $\mathrm{HCl} \mathrm{pH}$ 7.5.

7. $10 \mathrm{mM} \mathrm{MgCl} 210 \mathrm{mM}$ Tris- $\mathrm{HCl} \mathrm{pH} \mathrm{7.5.}$

8. Stock solution of $50 \mathrm{mg} / \mathrm{ml}(60.8 \mu \mathrm{M}$ ) rifampicin (weigh $0.5 \mathrm{~g}$ add $1.5 \mathrm{ml} \mathrm{HCl} \mathrm{0,5} \mathrm{N}$ slowly and fil to $10 \mathrm{ml}$ with water).

9. Stock solution of $20 \mathrm{mg} / \mathrm{ml}$ gentamycin sulphate in water.

10. Stock solution of $100 \mathrm{mg} / \mathrm{ml}(301 \mu \mathrm{M})$ spectinomycin in water.

\subsection{Other Reagents and Materials}

1. Half of a 0.5 litre plastic bottle (figure 3 ).

2. $1000 \mu \mathrm{L}$ pipette tip.

3. Needleless syringes.

4. Spectrophotometer measuring optic density at $600 \mathrm{~nm}$.

5. Microscope slide.

6. Coverslips.

7. Urgopore tape.

8. Scalpel or/and leaf tissue punch.

9. Microscope equipped with 488 or $515 \mathrm{~nm}$ lasers to excite mCIT; a $561 \mathrm{~nm}$ laser to excite $\mathrm{mCH}$ and a $445 \mathrm{~nm}$ laser to excite $\mathrm{mTU} 2$.

\subsection{Plasmids}

50 to $1000 \mu \mathrm{g} / \mathrm{ml}$ vectors compatible for agrobacteria transient expression in plant cell:

1. PI4P biosensor genetic construct in Gateway destination vector UBQ10prom::mCIT-P4M ${ }^{\text {SidM }}$ (Simon et al., 2016).

2. PI4P biosensor genetic construct in Gateway destination vector UBQ10prom::mCIT-PH ${ }^{\text {FAPPI- }}$ E50A (Simon et al., 2016).

3. Phosphatidylserine (PS) biosensor genetic construct in Gateway destination vector UBQ10prom::mCIT-C2 ${ }^{\text {Lact }}$ (Platre et al., 2018).

4. Your protein of interest to be tested for delocalisation upon PI4P depletion in fusion with a fluorescent protein cloned in a plant expression vector; for instance $p H 7 m 34 G W$. As an example in this protocol, we used UBQ10prom::2xmCH-KAI ${ }^{M A R K 1} / p H 7 m 34 G W$ (Platre et al., 2018). The system to deplete PI4P from the PM in Gateway destination vector: 2x35Sprom::MAP-mTU2-SAC1 $1_{2-518}$ (or 2x35Sprom::MAP-mCH-SAC1 $1_{2-518}$ )

and the corresponding mutated version to be used as negative control: 2x35Sprom::MAP-mTU2-SAC1 ${ }^{C 392 S}{ }_{2-518}$ (or 2x35Sprom::MAP-mCH-SAC1 ${ }^{C 392 S}{ }_{2-518}$ )

Briefly, MAP-mTU2 and MAP- $m C H$ were amplified to add the $12 \mathrm{~N}$-terminal residues (Myristoylation and Palmitoylation signal) of AtGPA1 (At2g26300) to mTURQUOISE2 using mTURQUOISE2/pEGFP-Cl as template (gift from Joachim Goedhart) or to mCHERRY using $m C H E R R Y / p D O N R 207$ as template. The resulting PCR products were cloned into pDONR221 by BP recombination to give MAP-mTU2noSTOP/pDONR221 and MAP-mCHnoSTOP/pDONR221 vectors. The catalytic domains of the $S$. cerevisiae Sac1p (AA 2 to 518 of Sac1p) and Sac1p ${ }^{\text {C392S }}$ (carrying a C392S mutation) were amplified using the PSEUDOJANIN (PJ) and PJ-INPP5E plasmids (gift of Gerald Hammond, University of Pittsburg, USA, addgene \#37999) and recombined into $p D O N R-P 2 R-P 3$ by BP recombination to give $S A C 1 / p D O N R-P 2 R-P 3$ and $S A C 1^{C 392 S} / p D O N R-P 2 R P 3$. Final destination vectors for 
agrobacteria transformation were obtained using LR recombination system (life technologies, http://www.thermofisher.com/) using $p K 7 m 34 G W$ (spectinomycin resistance for bacteria, kanamycin resistance in plants) destination vector for mTU2 constructs, and using pH7m34GW for $\mathrm{mCH}$ construts (spectinomycin resistance for bacteria, hygromycin resistance in plants)(Simon et al., 2016). (Note 1)

\section{Methods}

\subsection{Growth of Nicotiana benthamiana}

1. Sow N. benthamania seeds simply dropping them on a wet soil. For a week, keep the seedlings under half a plastic bottle to prevent dehydration (figure 3). Store seeds in a dark well ventilated location at $15-20^{\circ} \mathrm{C}$.

2. Between one and two weeks after sowing, prick out seedlings in individual pots. (Note 2)

\subsection{Transient transformation of Nicotiana benthamiana}

Following steps 1 to 3 should be conducted in sterile conditions. (Note 3)

1. Transform agrobacteria with each of the vectors of interest (see 2.4 and (Noack et al., 2019) for a detailed procedure).

2. Grow them in dark on LB agar plates supplemented with the appropriated antibiotics for $48 \mathrm{~h}$ at $29{ }^{\circ} \mathrm{C}$. Antibiotics should be used at the following concentrations for agrobacteria culture: rifampicin $50 \mu \mathrm{g} / \mathrm{ml}$; gentamycin $20 \mu \mathrm{g} / \mathrm{ml}$; spectinomycin $250 \mu \mathrm{g} / \mathrm{ml}$ (five times the concentration used for Escherichia coli). Alternatively, scoop few agrobacteria from a LB plate kept at $4{ }^{\circ} \mathrm{C}$ for few days or weeks, and spread it on a new one.

3. The day before agro-infiltration, use a pipette tip to scoop few colonies. Gently re-suspend them in $300 \mu \mathrm{L}$ of liquid LB by pipetting in and out several times.

4. Pour the re-suspended agrobacteria on a YEB agar plate supplemented with the appropriated antibiotics. Spread using sterile glass beads or a cell spreader, and let dry for $30 \mathrm{~min}$. Close the petri dish and incubate in dark for $16-24 \mathrm{~h}$ at $29^{\circ} \mathrm{C}$.

5. Using a $1000 \mu \mathrm{L}$ pipette tip, scoop the bacterial lawn and gently re-suspend in $1.5 \mathrm{ml}$ of $\mathrm{MgCl}_{2} 10 \mathrm{mM}$ Tris- $\mathrm{HCl} 10 \mathrm{mM} \mathrm{pH} 7.5$. Measure the optic density at $600 \mathrm{~nm}\left(\mathrm{OD}_{600}\right)$ obtained (Note 4). Dilute to the proper $\mathrm{OD}_{600}$.

6. Make premixes of agrobacteria containing the T-DNA to be co-expressed to a total $\mathrm{OD}_{600}=1$. For a co-infiltration of two types of agrobacteria, mix them so each has a final $\mathrm{OD}_{600}=0.5$.

(Note 5). Corresponding infiltration mixes could for instance correspond to:

- 2x35Sprom::MAP-mCH-SAC1 $2-518$ and UBQ10prom::mCIT-P4M ${ }^{\text {SidM }}$ (technical positive control).

- 2x35Sprom::MAP-mCH-SAC1 ${ }^{C 392 S}{ }_{2-518}$ and UBQ10prom::mCIT-P4M ${ }^{\text {SidM }}$ (technical negative control).

- 2x35Sprom::MAP-mCH-SACI $1_{2-518}$ and a construct encoding your protein of interest tagged with green, yellow, or blue fluorescent protein (here UBQ10prom::2xmCH-KAl ${ }^{M A R K I}$ ).

- 2x35Sprom::MAP-mCH-SAC1 ${ }^{C 392 S}{ }_{2-518}$ and a construct encoding your protein of interest tagged with green, yellow, or blue fluorescent protein (negative control).

For a co-infiltration of three types of agrobacteria, mix them so each has an $\mathrm{OD}_{600}=0.33$.

- 2x35Sprom::MAP-mTU2-SAC1 $1_{2-518}$ and UBQ10prom::mCIT-P4M ${ }^{\text {SidM }}$ together with your protein of interest (here $U B Q 10$ prom::2xmCH-KAI ${ }^{M A R K I}$ ). 
- 2x35Sprom::MAP-mTU2-SAC1 $2-518$ and UBQ10prom::mCIT-PH ${ }^{\text {FAPPI-E50A }}$ together with your protein of interest (here UBQ10prom::2xmCH-KAI ${ }^{\text {MARKI}}$ ).

- 2x35Sprom::MAP-mTU2-SAC1 $2-518$ and UBQ10prom::mCIT-C2 ${ }^{\text {Lact }}$ together with your protein of interest (here $U B Q 10$ prom: :2xmCH-KAI $I^{M A R K 1}$ ).

- 2x35Sprom::MAP-mTU2-SAC1 ${ }^{C 392 S}{ }_{2-518}$ and UBQ10prom::mCIT-P4M ${ }^{\text {SidM }}$ together with your protein of interest (here UBQ10prom::2xmCH-KAI ${ }^{M A R K l}$ ).

- 2x35Sprom::MAP-mTU2-SAC1 ${ }^{C 392 S}{ }_{2-518}$ and UBQ10prom::mCIT-PH ${ }^{\text {FAPPI-E50A }}$ together with your protein of interest (here UBQ10prom::2xmCH-KAI ${ }^{M A R K I}$ ).

- 2x35Sprom::MAP-mTU2-SAC1 ${ }^{C 392 S}{ }_{2-518}$ and UBQ10prom::mCIT-C2 ${ }^{\text {Lact }}$ together with your protein of interest (here UBQ10prom::2xmCH-KAI $1^{\text {MARKI }}$ ). (Note 6, 7)

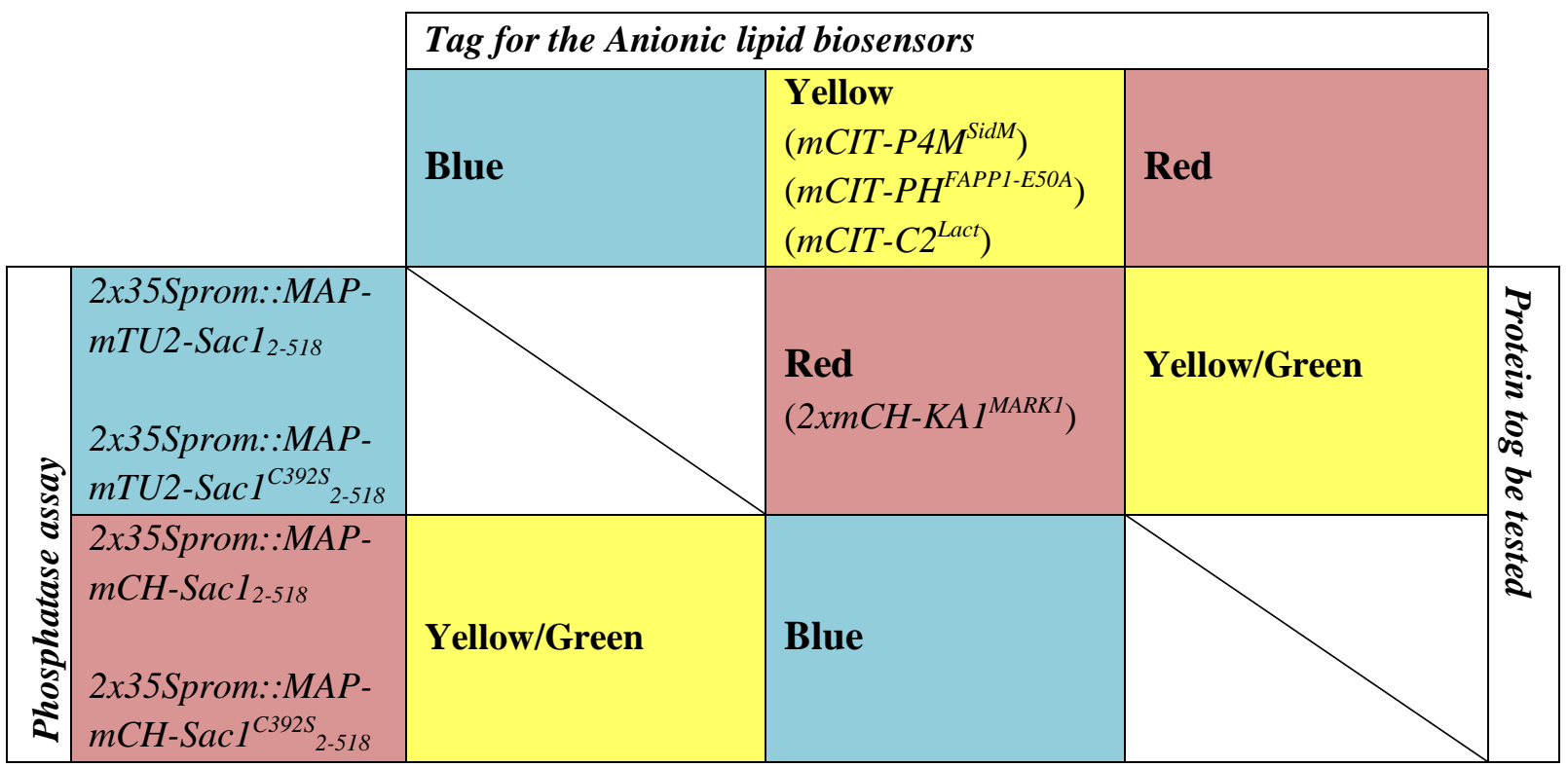

Table 1. Possible combinations for double or triple transformation of $N$. benthamiana. For Coinfiltration, anionic lipid biosensor (controls) and the protein of interest to be tested need to be transformed in different leaves, together with the 2x35Sprom::MAP-FP-SACl $1_{2-518}$ and the corresponding catalytic dead version. Transformed cells will express two constructions. For triple transformation of $N$. benthamiana, anionic lipid biosensor (controls) and the protein of interest need to be transformed in the same leave (transformed cells will co-express three constructions) together with the $2 x 35$ Sprom::MAP-FP-SAC1 $2-518$ and the corresponding catalytic dead version.

7. Use needleless syringes to infiltrate the abaxial side (dorsal side, facing the ground) of Nicotiana benthamiana leaves. (Note 8)

8. Bring back N. benthamiana plants to the greenhouse or growth chamber. (Note 9)

9. Perform imaging $36 \mathrm{~h}$ after agroinfiltration. (Note 10, 11)

\subsection{Imaging using fluorescence microscope}

1. Add a droplet of water or $1 \frac{1}{2} \mathrm{MS}$ on a microscope slide.

2. Cut a piece of agro-infiltrated leaves using a scalpel or a leaf tissue punch.

3. Put it on the coverlide upside-down: the abaxial side facing up.

4. Add another droplet of water or $1 / 2 \mathrm{MS}$ on the piece of leaf, and cover with a coverslip.

5. Gently tether the coverslip to the slide with urgopore tape.

6. Image using a confocal fluorescence microscope. 
7. Start by visualizing MAP-mTU2-Sac1 ${ }^{\mathrm{C} 492 \mathrm{~S}}{ }_{2-518}$ at the plasma membrane of leaf pavement cell (using $445 \mathrm{~nm}$ laser excitation). In this condition, both the PI4P biosensor mCIT-P4M ${ }^{\mathrm{SidM}}$ or mCIT-PH ${ }^{\text {FAPP1-E50A }}$ or negative control UBQ10prom::mCIT-C2 ${ }^{\text {Lact }}$ (using a 488/515 nm laser excitation) and your protein of interest (using red channel) should be localised at the plasma membrane of pavement cells (figure 2). (Note 12)

8. Identify and image cells in which MAP-mTU2-Sac12-518 (using $445 \mathrm{~nm}$ laser excitation) is at the plasma membrane of leaf pavement cell. In cells expressing MAP-mTU2-Sac1 $2-518$, the PI4P biosensor mCITRINE-P4M ${ }^{\text {SidM }}$ or mCITRINE-1 $x$ PH $^{\text {FAPP1-E50A }}$ should be re-localised to endosomes where the minor pool of PI4P sits in plant cells (figure 2). (Note 13)

9. In cells expressing MAP-mTU2-Sac1 $1_{2-518}$, no effect should be observed for the PS biosensor UBQ10prom::mCIT-C2 ${ }^{\text {Lact }}$ that should still be observed at the plasma membrane. (Note 14, $15,16)$

10. Assess the effect of the depletion of PI4P on the localisation of your protein of interest by imaging the corresponding fluorescence. For example when coinfiltrated together with MAPmTU2-Sac1 $1_{2-518,}$ 2 $\mathrm{mmCH}-\mathrm{KA} 1^{\mathrm{MARK} 1}$ relocalised in the cytoplasm (figure $2 \mathrm{E}, \mathrm{F}$ and $\mathrm{G}$ ). (Note 17)

\subsection{Quantification}

1. For quantification, only consider cells that have been successfully transformed with all constructs agro-infiltrated.

2. Count the proportion of cells displaying regular localisation of the protein of interest when coexpressed with MAP-FP-Sac1 $1_{2-518}$ and MAP-FP-Sac1 ${ }^{\mathrm{C} 392 \mathrm{~S}}{ }_{2-518}$.

3. In case of triple transformation, you can as well restrict the sampling to cells where the PI4P sensor is efficiently delocalised (figure $2 \mathrm{H}$ ). In case of double transformation, make sure to also quantify the effect of MAP-FP-Sac1 $1_{1-518}$ and MAP-FP-Sac1 ${ }^{\mathrm{C} 392 \mathrm{~S}}{ }_{2-518}$ on PI4P sensor subcellular localisation (figure 2I). (Note 18)

4. Perform a statistical analysis on the data obtained using an exact Fisher test (suited for small sample size) or a Chi-squared test. In both cases, assess the dependency of the number of cells displaying normal or relocalised distribution of the protein of interest when co-expressed together with MAP-FP-Sac1 ${ }_{1-518}$ and MAP-FP-Sac1 ${ }^{\mathrm{C} 392 \mathrm{~S}}{ }_{2-518}$. For instance, if having (i) 25 cells co-expressing MP-mCH-Sac1 ${ }^{\mathrm{C} 392 \mathrm{~S}}{ }_{2-518}$ and the protein of interest, among which 22 display a normal subcellular localisation of the protein of interest at the PM and/or endosomes, and (ii) 31 cells co-expressing MP-mCH-Sac1 $1_{2-518}$ and the protein of interest among which 23 display a relocalised protein of interest; then the corresponding R-commands would be fisher.test(matrix $(\mathrm{c}(22,3,8,23), 2,2, \quad$ byrow=TRUE) $)$ and chisq.test(matrix $(\mathrm{c}(22,3,8,23), 2,2$, byrow=TRUE), correct=FALSE). Make sure to sample at least 15 cells per condition. The experiment should be independently replicated three times.

\section{Notes}

Note 1: Genetic constructs agro-infiltrated must bear a promoter expressed in Nicotiana benthamiana leaf epidermis and strong enough.

Note 2: 4 weeks-old plants generally are fine for agroinfiltration. Do not use plants that started to produce flower. 
Note 3: The agroinfiltration protocol described does not require acetosyringone.

Note 4: Be extra careful when resuspending agrobacteria in $\mathrm{MgCl}_{2}$-Tris solution, for instance avoid vortexing.

Note 5: OD 600 generally evolves linearly with particle (bacteria) concentration between 0.2 and 1 . Therefore, it is necessary to dilute the agrobacteria resuspended prior to assess OD $_{600}$. In our conditions, when resuspending the content of a petri plate in $2 \mathrm{ml}$ of $\mathrm{MgCl}, 1: 40 \mathrm{v} / \mathrm{v}$ dilution allowed to reach $O D_{600}$ comprised between 0.2 and 1 .

Note 6: you will need more of the culture for the protein you want to test for delocalisation upon PI4P depletion (here UBQ10prom::2xmCH-KAI MARK1) than for the other constructs.

Note 7: 2x35Sprom::MAP-mCH-SAC1 $2-518$ and 2x35Sprom::MAP-mCH-SAC1 ${ }^{C 392 S}{ }_{2-518}$ can be used instead of the mTU2 constructs. In this case, either used a blue-tagged protein of interest and perform a triple transformation. Alternatively, use a green/yellow tagged protein of interest and perform double transformations.

Note 8: Carefully chose the leaves to agro-infiltrate. Flat heart-like shaped are the best, rather than roundish that are too old, or than small wavy leaves that are too young. Also, it is wise to agroinfiltrate two leaves with the same premix and to image both to reduce variability.

Note 9: Agrobacteria are light sensitive. Therefore, infiltrated Nicotiana benthamiana can be kept for few hours in a shadowed location, and not taken right away in a growth chamber or greenhouse.

Note 10: When co-infiltrating two constructs, using final $O D_{600}=1$ for the mix, we generally achieved 60 to $90 \%$ co-transformed epidermal cells.

Note 11: Do not exceed a 48 h-window after agro-infiltration to image the leaves. Indeed, using strong or mild promoters, proteins then start to adopt irrelevant localisations, possibly because they aggregate.

Note 12: if using MAP-mCH-Sac1 ${ }_{2-518,}^{\mathrm{C} 392 \mathrm{~s} e} 561 \mathrm{~nm}$ laser excitation.

Note 13: Upon depletion of PI4P from the PM, only some endomembranes comprise PI4P. The corresponding organelles are fast moving compartments in $N$. benthamiana epidermal cells, and therefore a spinning disc microscope may be better suited than a scanning confocal microscope.

Note 14: mCITRINE-P4M SidM sometimes also, or instead, solubilises into the cytoplasm as fluorescence is seen in cytoplasmic threads and surrounding intracellular compartments (figure $1 E, F$ and $H$ ). The expression level may cause the here before mentioned discrepancy: the stronger the expression, the more cytosolic the localisation (data not shown).

Note 15: use similar confocal settings when comparing fluorescence intensity or for quantification.

Note 16: For quantitative imaging, pictures of epidermal root cells were taken with detector settings optimized for low background and no pixel saturation. 
Note 17: you can have a mosaic expression therefore you should covisualised MAP-mTU2-Sac1 1-511 or MAP-mCH-Sac1 $1_{1-511}$ together with your protein of interest for subsequent quantification

Note18: As blue fluorescence corresponding to MAP-mTU2-Sac1 2-518 and MAP-mTU2-Sac1 ${ }^{\text {C392S }}{ }_{2-512}$ recombinant proteins might prove hard to image, a general quantification can also be perform. However, it requires the transformation efficiency to be high enough (>75\%) (Figure 2.I).

\section{Acknowledgments}

We thank Yvon Jaillais (RDP laboratory, ENS LYON, France) for comments on the manuscript, as well as Romain Boisseau (OBEE department, University of Montana, USA) and Antoine Grissot (Department of Vertebrate Ecology and Zoology, University of Gdansk, Poland) for their advice concerning the statistical analysis. This work was supported by Seed Fund ENS LYON-2016 and Junior Investigator grant ANR-16-CE13-0021. M.D. is funded by a fellowship from the French Ministry of Higher Education.

\section{Figure Legends}



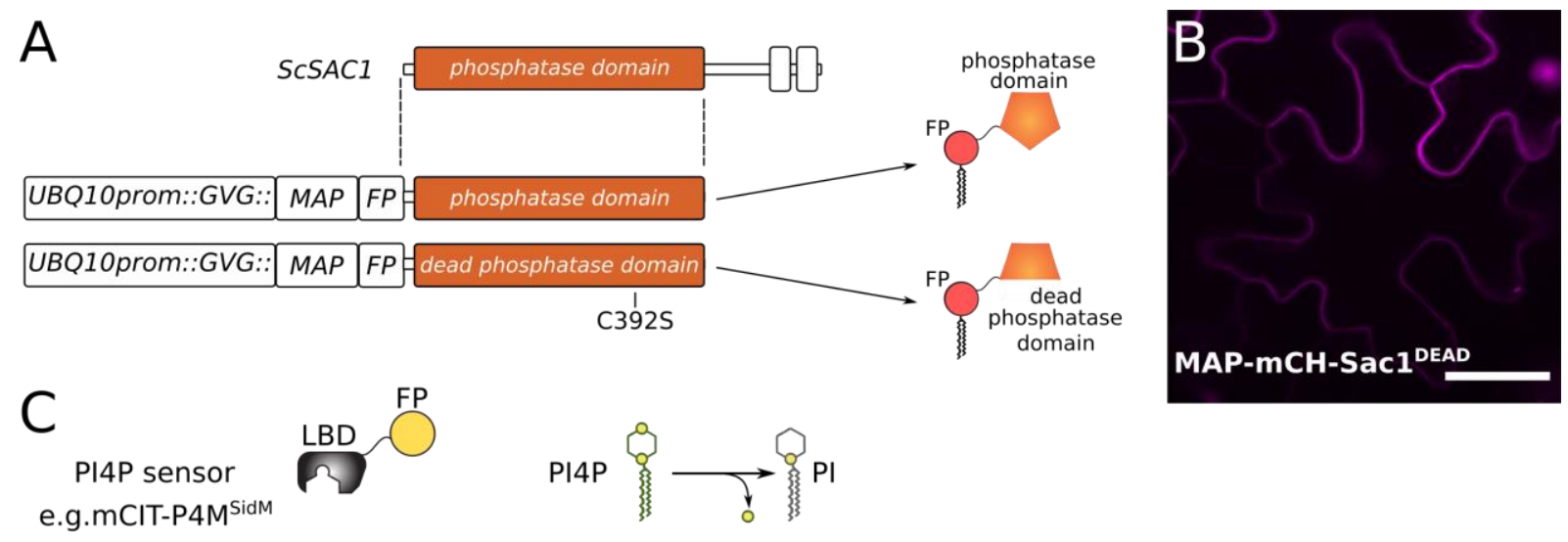

D
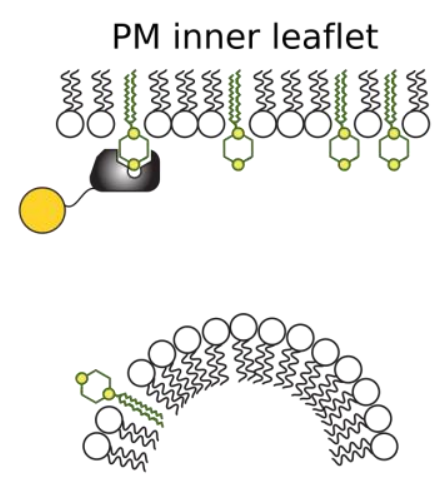

endosome outer leaflet
$E$ PM inner leaflet
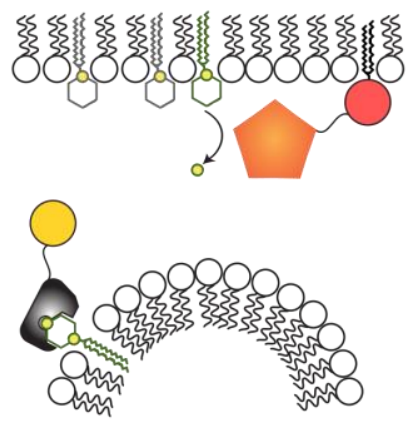

endosome outer leaflet
$\mathrm{F}$

PM inner leaflet
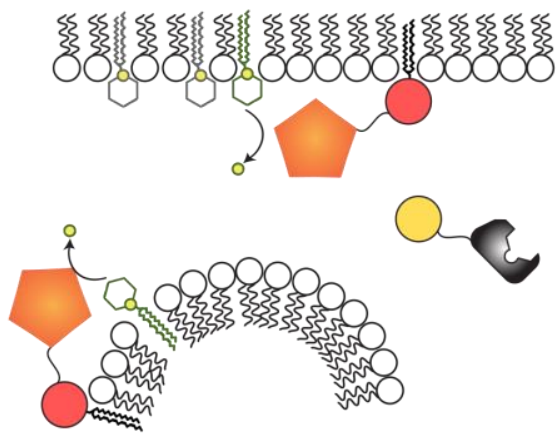

endosome outer leaflet
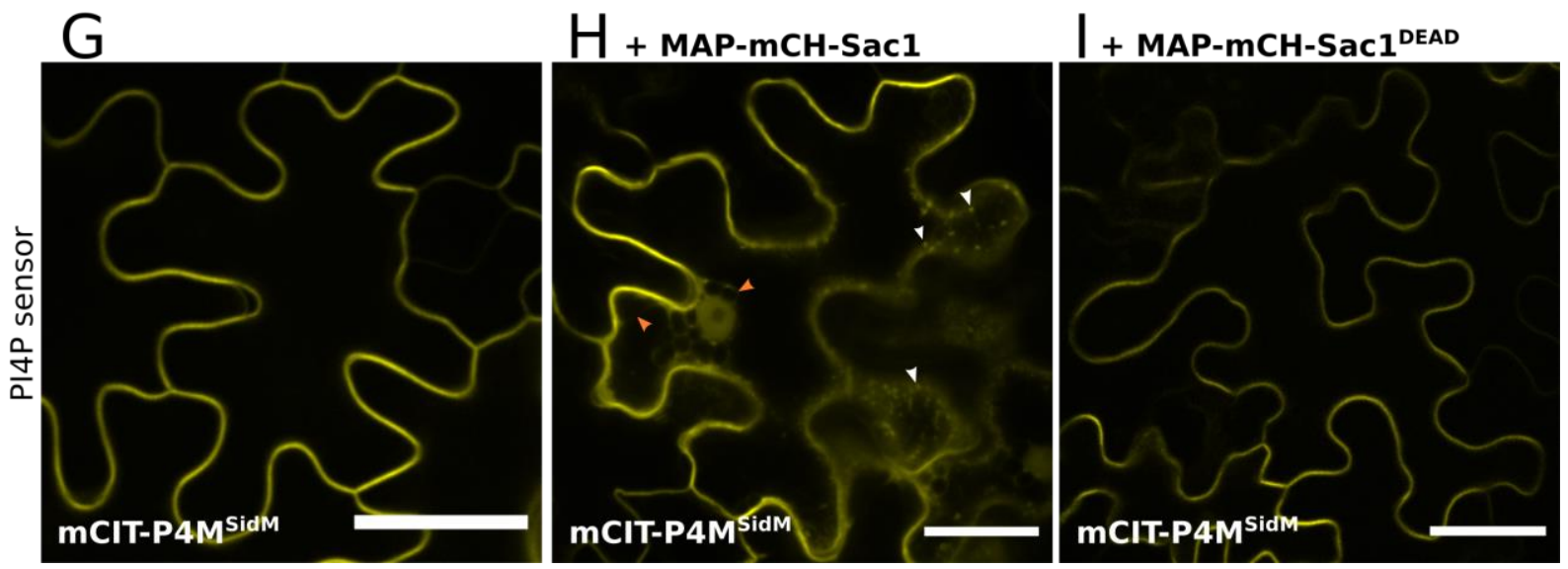

Figure 1. MAP-FP-Sac1 $1_{2-518}$ efficiently depletes the plant cell in PI4P. (A) Recombinant genetic constructs used to dephosphorylate PI4P at the plasma membrane, and possibly to a lesser extent at endosomes (see abbreviation section). (B) MAP-FP-Sac ${ }^{\mathrm{C} 392 \mathrm{~S}}{ }_{2-518}$ is targeted at the plasma membrane when expressed in $N$. benthamiana. (C) PI4P sensors are composed of a lipid binding domain fused to a fluorescent protein. PI4P sensors, e.g. mCIT-P4M $\mathrm{M}^{\mathrm{SidM}}$, specifically binds PI4P, but not PI. (D) In resting conditions, PI4P sensors only (mCIT-P4M $\left.{ }^{\mathrm{SidM}}\right)$ or mainly $\left(\mathrm{mCIT}^{\mathrm{P}} \mathrm{PH}^{\mathrm{FAPP1}-\mathrm{E} 50 \mathrm{~A}}\right)$ label the PM, which comprises the major pool of PI4P in plant cells. (E) Upon PI4P depletion at the PM, PI4P biosensors are expected to relocalise to endosomes at which membranes the minor pool of PI4P sits. (F) PI4P depletion may also solubilise PI4P sensors into the cytoplasm. (G) mCIT-P4M ${ }^{\text {SidM }}$ localises at the PM when infiltrated alone (corresponds to G scheme). (H) In cells expressing MAP-mCH$\mathrm{Sac1}_{2-518}, \mathrm{mCIT}-\mathrm{P} 4 \mathrm{M}^{\mathrm{SidM}}$ relocalises to endosomes (white arrowhead) and is seen in cytoplasmic threads and around large organelles (orange arrowhead), showing it is solubilised in the cytosol 
(corresponds to $\mathrm{E}$ and $\mathrm{F}$ schemes). (I) On the other hand, mCIT-P4M ${ }^{\text {SidM }}$ localisation is not affected by the expression of MAP-mCH-Sac1 ${ }^{\mathrm{C} 392 \mathrm{~S} 2}{ }_{2-518}$. Scale bars: $40 \mu \mathrm{m}$. 
A
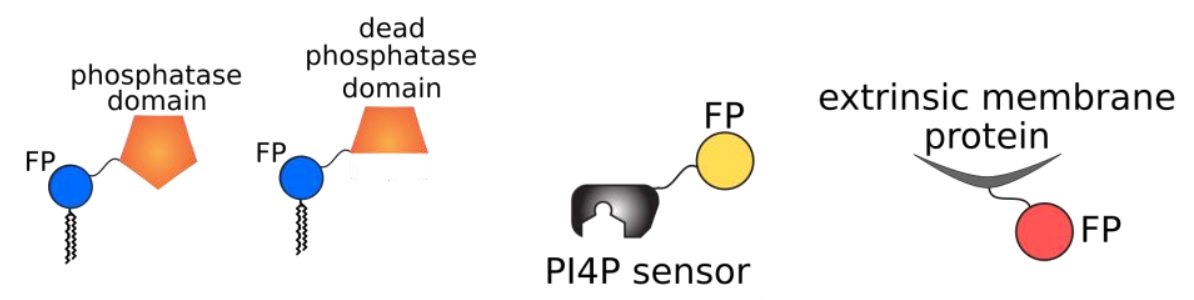

e.g.mCIT-P4M ${ }^{\text {SidM }}$

B PM inner leaflet
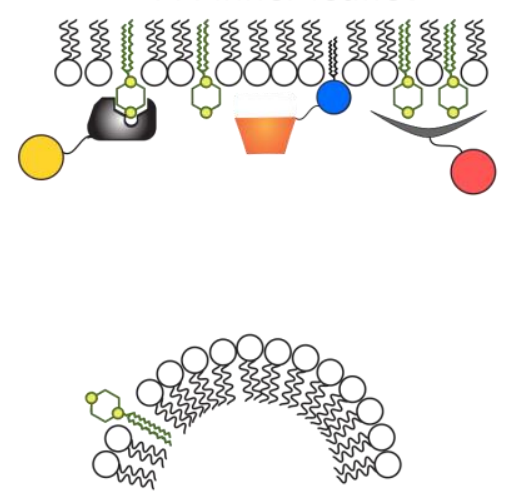

endosome outer leaflet

E

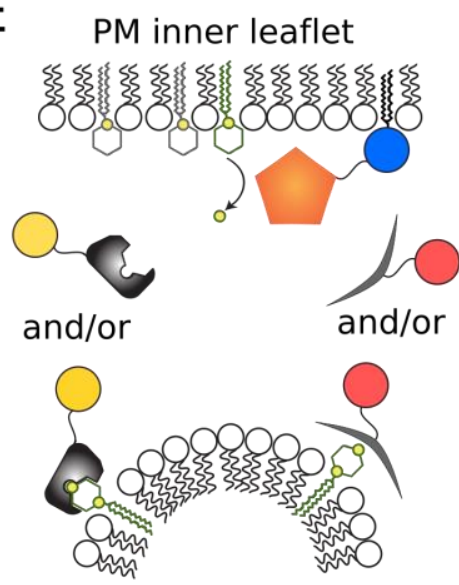

endosome outer leaflet

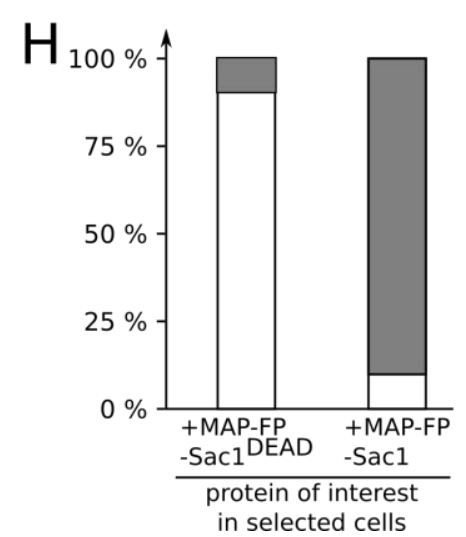

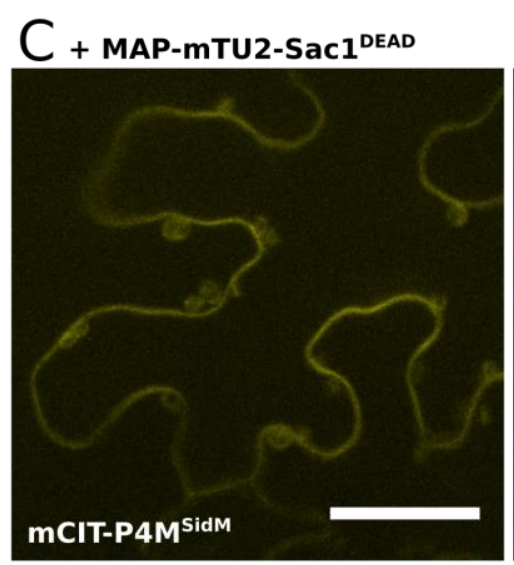
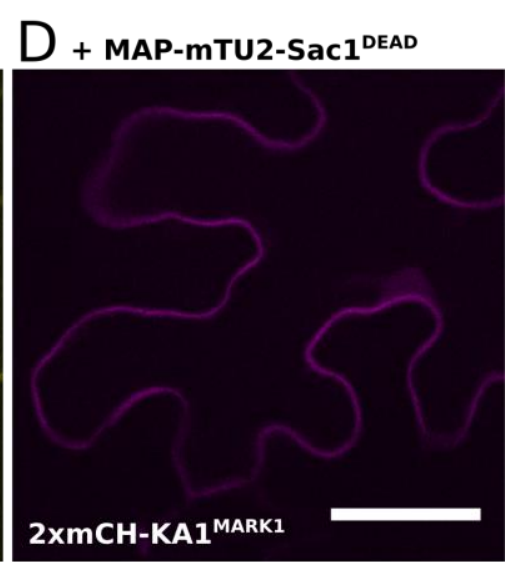

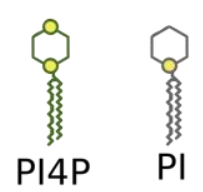

PI4P

\section{(1)}


both PI4P sensor and a protein relying on PI4P for its localisation are properly localised. (E-G) Upon expression of MAP-FP-Sac1 $1_{1-511}$, both mCIT-P4M $^{\text {SidM }}$ and $2 \mathrm{xmCH}^{-K A 1}{ }^{\mathrm{MARK} 1}$ display a new subcellular localisation. They are enriched in intracellular compartments (white arrowheads) and/or solubilised in the cytosol as indicated by cytoplasmic threads and surroundings of large organelles (orange arrowheads). (H and I) Example of quantification. Scale bars: $40 \mu \mathrm{m}$.

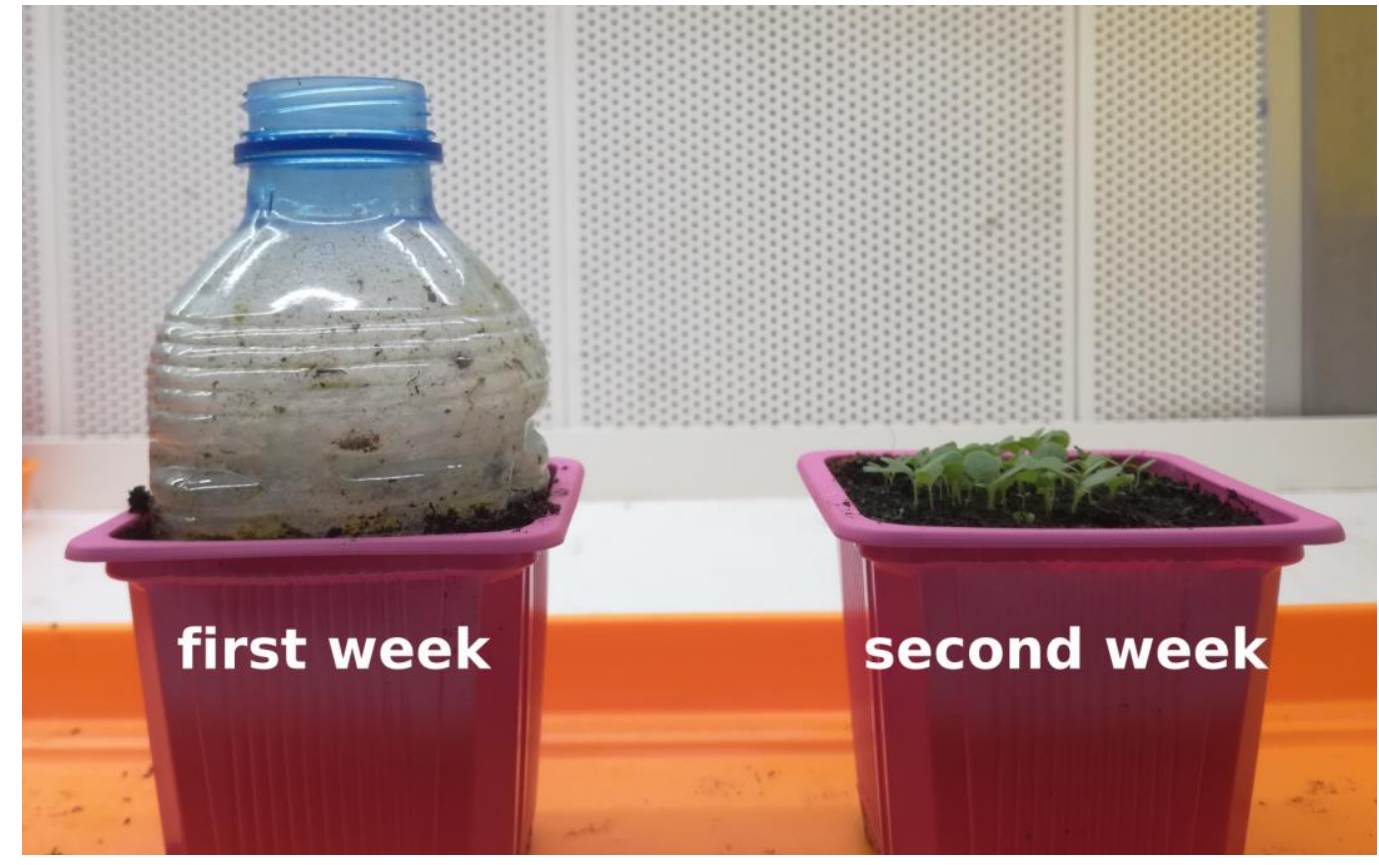

Figure 3.

First two weeks of culture of Nicotiana benthamiana. Seeds are sowed by simply dropping them onto soil. Seedlings sprout under a cut bottle to prevent dehydration (left). The week after, the bottle is removed (right) and seedlings can be pricked out into individual pots.

\section{Index}

2x35Sprom: strong and ubiquitous viral promoter.

2xmCH: two CHERRY, red fluorescent proteins.

FP: fluorescent protein.

MAP: myristoylation and palmitoylation sequence (Simon et al., 2016)

mCIT: monomeric CITRINE, an enhanced YFP.

mTU2: monomeric TURQUOISE2, a blue fluorescent protein.

PI: phosphatidulinositol.

PI4P: phosphatidylinositol 4-phosphate.

PM: plasma membrane.

PS: phosphatidylserine.

Sac1: Sac12-518.

Sac1 ${ }^{\text {DEAD: }}$ Sac1 $^{\text {C392S }}{ }_{2-518}$.

ScSACl or SACl: Saccharomyces cerevisiae gene encoding Sac1p protein.

UBQ10prom: mild and ubiquitous promoter from Arabidopsis thaliana UBIQUITIN10 gene.

\section{References}


Brombacher E, Urwyler S, Ragaz C, et al., 2009. Rab1 guanine nucleotide exchange factor SidM is a major phosphatidylinositol 4-phosphate-binding effector protein of Legionella pneumophila. J Biol Chem 284, 4846-56.

Del Campo CM, Mishra AK, Wang YH, Roy CR, Janmey PA, Lambright DG, 2014. Structural basis for PI(4)P-specific membrane recruitment of the Legionella pneumophila effector DrrA/SidM. Structure 22, 397-408.

Guo S, Stolz LE, Lemrow SM, York JD, 1999. SAC1-like domains of yeast SAC1, INP52, and INP53 and of human synaptojanin encode polyphosphoinositide phosphatases. J Biol Chem 274, 12990-5.

Hammond GR, Machner MP, Balla T, 2014. A novel probe for phosphatidylinositol 4phosphate reveals multiple pools beyond the Golgi. J Cell Biol 205, 113-26.

Lemmon MA, 2008. Membrane recognition by phospholipid-binding domains. Nat Rev Mol Cell Biol 9, 99-111.

Monnard PA, Walde P, 2015. Current Ideas about Prebiological Compartmentalization. Life (Basel) 5, 1239-63.

Moravcevic K, Mendrola JM, Schmitz KR, et al., 2010. Kinase associated-1 domains drive MARK/PAR1 kinases to membrane targets by binding acidic phospholipids. Cell 143, 96677.

Noack LC, Jaillais Y, 2017. Precision targeting by phosphoinositides: how PIs direct endomembrane trafficking in plants. Curr Opin Plant Biol 40, 22-33.

Noack LC, Pejchar P, Sekeres J, Jaillais Y, Potocky M, 2019. Transient Gene Expression as a Tool to Monitor and Manipulate the Levels of Acidic Phospholipids in Plant Cells. Methods Mol Biol 1992, 189-99.

Platre MP, Jaillais Y, 2017. Anionic lipids and the maintenance of membrane electrostatics in eukaryotes. Plant Signal Behav 12, e1282022.

Platre MP, Noack LC, Doumane M, et al., 2018. A Combinatorial Lipid Code Shapes the Electrostatic Landscape of Plant Endomembranes. Dev Cell 45, 465-80 e11.

Simon ML, Platre MP, Assil S, et al., 2014. A multi-colour/multi-affinity marker set to visualize phosphoinositide dynamics in Arabidopsis. Plant J 77, 322-37.

Simon ML, Platre MP, Marques-Bueno MM, et al., 2016. A PtdIns(4)P-driven electrostatic field controls cell membrane identity and signalling in plants. Nat Plants 2, 16089.

Zhu Y, Hu L, Zhou Y, Yao Q, Liu L, Shao F, 2010. Structural mechanism of host Rab1 activation by the bifunctional Legionella type IV effector SidM/DrrA. Proc Natl Acad Sci U S A 107, 4699-704. 